\title{
Un modelo trófico preliminar del ecosistema pelágico del norte de Chile (18 $\left.20^{\circ} \mathrm{S}-24^{\circ} 00^{\prime} \mathrm{S}\right)$
}

\author{
Marianela Medina $^{1}$, Hugo Arancibia ${ }^{2}$ \& Sergio Neira ${ }^{3}$ \\ ${ }^{1}$ Departamento Ciencias del Mar, Universidad Arturo Prat. Iquique, Chile \\ ${ }^{2}$ Facultad de Ciencias Naturales y Oceanográficas, Universidad de Concepción. Concepción, Chile \\ ${ }^{3}$ Zoology Deparment, University of Cape Town, Cape Town, Republic of South Africa
}

\begin{abstract}
RESUMEN. Se construye un modelo ecotrófico con balance de masa para cuantificar los flujos de energía/materia e interacciones tróficas en la trama trófica del ecosistema pelágico del norte de Chile, en el año 1989. El modelo incluye 13 grupos funcionales, siendo la mayoría de ellos recursos pesqueros, aunque considera especies de la fauna acompañante de la pesquería industrial de cerco, fitoplancton, zooplancton, detritos y predadores tope. Se utiliza el modelo y paquete computacional Ecopath con Ecosim versión 5.1. Los parámetros de entrada para el área de estudio (18²0'S a $24^{\circ} 00^{\prime}$ 'S y desde la costa hasta $60 \mathrm{mn}$ al oeste, equivalente a $35.000 \mathrm{~km}^{2}$ ) se obtuvieron desde literatura especializada y desde otros modelos aplicados a ecosistemas de surgencia. Los resultados indican que los flujos totales, que son una estimación del tamaño del ecosistema, alcanzan a 38.674 ton $\mathrm{km}^{-2} \cdot \mathrm{año}^{-1}$, mientras que la producción primaria estimada se calcula en 13.452 ton $\mathrm{km}^{-2}$ año ${ }^{-1}$. El nivel trófico promedio de la pesquería del norte de Chile fue de 2,7, lo que refleja que las capturas se sostienen en especies de niveles trófico bajo, principalmente sardina (Sardinops sagax). La eficiencia de transferencia media de energía fue de $9,8 \%$, valor que está dentro del rango característico de $10 \%$ calculado para ecosistemas marinos. Este ecosistema puede caracterizarse como un sistema que está alejado de la madurez, baja eficiencia de transferencia de energía, cadenas tróficas cortas y un bajo reciclaje de materia, lo que es consistente con lo informado para los principales ecosistemas de surgencias del mundo.
\end{abstract}

Palabras clave: modelo ecotrófico, interacciones tróficas, flujo de energía, ecosistema de surgencia, norte de Chile.

\section{A preliminary trophic model of the pelagic ecosystem in northern Chile} $\left(18^{\circ} 20^{\prime} \mathrm{S}-24^{\circ} 00 ' S\right)$

\begin{abstract}
An ecotrophic model was constructed to assess energy flows in the pelagic food web off northern Chile $\left(18^{\circ} 20^{\prime} \mathrm{S}-24^{\circ} 00^{\prime} \mathrm{S}\right.$; from the coast to $60 \mathrm{~nm}$ west; encompassing $\left.35,000 \mathrm{~km}^{2}\right)$ in 1989 . Required input parameters were obtained from the literature and other models applied to upwelling ecosystems. The model considered 13 functional groups. Although the analysis emphasized fishery resources, it also included by-catch species from the pelagic fishery and other important groups such as phytoplankton, zooplankton, detritus, and top predators. The model was built using Ecopath with Ecosim software version 5.1. The results indicated that the total flow, an estimate of system size, was 38,674 ton $\cdot \mathrm{km}^{-2} \cdot \mathrm{year}^{-1}$. System primary production was calculated at 13,452 ton $\cdot \mathrm{km}^{-2} \cdot$ year $^{-1}$. The average trophic level of the catch in northern Chile is estimated to be 2.7 , indicating that catches are sustained mainly by species from lower trophic levels, such as sardine (Sardinops sagax). The average transfer efficiency is estimated to be $9.8 \%$, which is in the range reported for marine food webs. This ecosystem is far from maturity, has low-efficiency energy transfers, short trophic chains, and low levels of recycled matter, consistent with reports from the world's main upwelling ecosystems.
\end{abstract}

Key words: trophic model, trophic interactions, energy flow, upwelling ecosystem, northern Chile.

Autor corresponsal: Marianela Medina (marianela.medina@unap.cl).

\section{INTRODUCCIÓN}

El creciente interés en la modelación de ecosistemas marinos y sus pesquerías ha permitido identificar los componentes bióticos y abióticos que juegan roles importantes en términos de la estructura y dinámica comunitaria. Específicamente, el interés de investigadores y administradores de recursos marinos se ha dirigido hacia la identificación de las interacciones predador-presa que regulan la 
distribución y abundancia de los organismos vivos, más allá de la mera evaluación de la biomasa de los recursos de importancia comercial. Actualmente, el paradigma del enfoque ecosistémico aplicado a pesquerías impone la necesidad de comprender los efectos de la pesquería en el ecosistema y los efectos del ecosistema en las pesquerías (Mackinson et al., 1997; Rice 2000; Shannon et al., 2000).

Los modelos de balance de masa representan un enfoque simple para el análisis de interacciones tróficas en sistemas con recursos pesqueros. Dentro de estos, el enfoque Ecopath propuesto por Polovina (1984) y desarrollado posteriormente por Christensen \& Pauly (1992) y Walters et al. (1997), ha sido ampliamente aplicado a ecosistemas acuáticos alrededor del mundo, como por ejemplo, en el ecosistema pelágico del Océano Pacífico Oriental Tropical (Olson \& Watters, 2003), en el ecosistema de las pesquerías pelágicas del Perú (Jarre et al., 1991), quienes modelan las fluctuaciones del ecosistema en tres periodos: cuando la anchoveta fue moderadamente abundante, cuando la biomasa de la anchoveta y el esfuerzo de pesca fueron altos, y finalmente el periodo del colapso de la pesquería. Ecopath también ha sido ampliamente aplicado en el ecosistema de surgencia al norte de Benguela (i.e. Shannon \& Jarre-Teichmann, 1999; Shannon et al., 2000), donde se le considera una herramienta necesaria para la implementación del enfoque ecosistémico en el manejo de pesquerías.

En Chile, Ecopath ha sido aplicado al sistema de surgencia de la bahía de Tongoy en el norte de Chile (Wolff, 1994). Recientemente, Arancibia et al. (2003), Neira \& Arancibia (2004) y Neira et al. (2004), modelan la trama trófica en el ecosistema marino de Chile central $\left(33^{\circ} \mathrm{S}-39^{\circ} \mathrm{S}\right)$, explorando interacciones tróficas entre los principales recursos pesqueros, sus predadores y presas, y comparan, por ejemplo, la estructura comunitaria antes y después de eventos El Niño.

El ecosistema pelágico del norte de Chile, desde Arica ( $\left.18^{\circ} 21^{\prime} \mathrm{S}\right)$ hasta Antofagasta $\left(24^{\circ} \mathrm{S}\right)$ se encuentra influenciado por el sistema de corrientes de ChilePerú, que es uno de los más productivos del mundo debido a la acción combinada de las surgencias costeras y del transporte de nutrientes subantárticos (Bernal et al., 1990; Pizarro et al., 1994). Históricamente, este sistema ha sustentado una relevante pesquería pelágica compuesta, en orden de importancia en los desembarques, por anchoveta (Engraulis ringens), sardina (Sardinops sagax), jurel (Trachurus symmetricus), caballa (Scomber japonicus) y como fauna acompañante, bonito (Sarda chilensis) y cojinoba del norte (Seriolella violacea), principalmente, con fuertes variaciones en sus desembarques a través del tiempo debido a factores oceanográficos como también a la presión pesquera.

La necesidad de tomar en cuenta la estructura y función del ecosistema, y en particular la interacción entre las especies explotadas y su ambiente (biótico y abiótico) en la administración de recursos marinos, es ampliamente reconocida. Esto motiva el desarrollo de, entre otras herramientas, modelos tróficos ecosistémicos que pudieran ser utilizados como un complemento a los modelos monoespecíficos clásicos, permitiendo una evaluación y manejo de los recursos pesqueros bajo un enfoque holístico (Escobar, 2001; Ortiz \& Wolff, 2002; Olson \& Watters, 2003).

Por lo expuesto anteriormente, el objetivo de este estudio es construir un modelo ecotrófico utilizando el programa Ecopath con Ecosim (EwE) para cuantificar y describir interacciones biológicas y flujos de energía entre los principales componentes del ecosistema pelágico del norte de Chile.

\section{MATERIALES Y MÉTODOS}

\section{Área y periodo de estudio}

Para efectos de este estudio, se delimitó el área de estudio desde los $18^{\circ} 20^{\prime} \mathrm{S}$ hasta los $24^{\circ} 00^{\prime} \mathrm{S}$ y desde la costa hasta las $60 \mathrm{mn}$ al oeste, abarcando unos $35.059 \mathrm{~km}^{2}$ (Fig. 1). Los criterios utilizados son: i) es la zona donde opera la flota industrial de cerco y ii) que es el área de influencia más importante de la productividad asociada a la surgencia costera.

El periodo de estudio comprende el año 1989 considerando que el ecosistema marino de la zona norte se encontraba en una condición estado-estable esto es, que las entradas al sistema son iguales a las salidas (sensu Christensen \& Pauly, 1993) y que las poblaciones se encontraban en equilibrio (sensu Allen, 1971). Antes de 1989 y después de 1990 se presentaron dos eventos El Niño en la zona norte de Chile, estos fueron El Niño 1986-1987 y El Niño 1991-1992 (Fuenzalida, 1992).

El modelo consideró 13 grupos funcionales, siendo la mayoría recursos pesqueros y otros pertenecientes a la fauna acompañante de la pesquería industrial de cerco. Estos son: anchoveta (Engraulis ringens), sardina (Sardinops sagax), jurel (Trachurus symmetricus), caballa (Scomber japonicus), cojinoba 


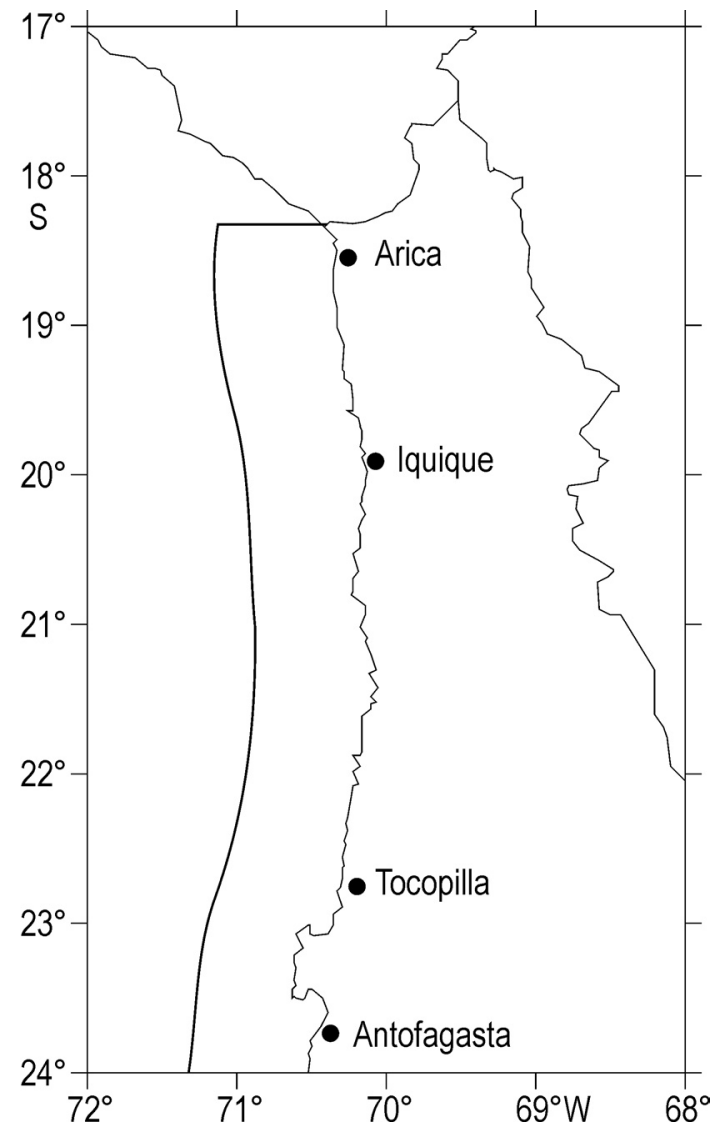

Figura 1. Área de estudio en la zona norte de Chile (18 20 'S-2400'S).

Figure 1. The study area, northern Chile $\left(1^{\circ} 20\right.$ 'S2400'S).

(Seriolella violacea), bonito (Sarda chilensis), tiburones (Isurus oxyrinchus y Prionace glauca) siendo estas especies recursos importantes de la pesquería artesanal entre Arica y Antofagasta (Fig. 1), lobo marino común (Otaria flavescens), aves marinas tales como cormorán (Phalacrocorax bougainvillii) y pelícano (Pelecanus thagus), peces mesopelágicos, zooplancton, fitoplancton y detritos.

\section{El modelo Ecopath}

El modelo Ecopath (Polovina, 1984; Christensen \& Pauly, 1992) fue utilizado para cuantificar las interacciones tróficas entre los grupos funcionales del área estudiada. Ecopath brinda un marco para la construcción de modelos de tramas tróficas con balance de masa. Se genera el balance de masa a partir de estimaciones de la abundancia de los grupos (biomasa), la tasa de productividad o mortalidad de los mismos, cómo interactúan (composiciones de dieta y tasas de consumo de alimento) y la eficacia con la cual se utilizan los recursos en el ecosistema.

El modelo entrega un cuadro estático de la estructura trófica de un ecosistema usando un sistema de ecuaciones lineales simultáneas, una para cada especie o grupo $i$ del sistema, para modelar el destino de la producción de cada grupo, la que es equilibrada por la depredación por otros componentes del sistema (mortalidad por depredación), sus exportaciones (mortalidad por pesca más otras exportaciones) y otras mortalidades (aquellas independientes de la depredación y capturas). La ecuación básica es la siguiente:

Producción de $i=$ toda la depredación sobre $i+$ pérdidas de biomasa por no depredación sobre $i+$ capturas de $i+$ otras exportaciones de $i$.

En términos matemáticos, esta ecuación corresponde a:

$$
B_{i} *\left(P_{i} / B_{i}\right) * E E_{i}-\sum_{j=1}^{n}\left(B_{j} *(Q / B)_{j} * D C_{i j}\right)-E X_{i}=0
$$

donde $i$ indica un componente del modelo, $j$ indica cualquiera de los predadores de $i, \mathrm{~B}_{i}$ es la biomasa de $i,(\mathrm{P} / \mathrm{B}) i$ es la producción de $i$ por unidad de biomasa el cual es equivalente al coeficiente de mortalidad total $(Z)$ en condiciones estado-estacionarias (Allen, 1971), (Q/B) $i$ es el consumo de $i$ por unidad de biomasa, DC $i j$ indica la fracción de $i$ en la dieta de $j$ (en términos de masa), EE $i$ es la eficiencia ecotrófica de $i$ (la fracción total de la producción que es consumida por predadores o exportada desde el sistema), EX $i$ representa las exportaciones de $i$ (por emigración o capturas pesqueras) (Christensen \& Pauly, 1992).

El balance de energía de cada componente está dado por:

$$
Q=P-R-U
$$

donde $Q$ es consumo que corresponde al consumo de presas tanto dentro del sistema como de presas halóctonas (importaciones); $P$ es producción, la que debe ser consumida por predadores, ser exportada desde el sistema o ser una contribución al detrito; $R$ es respiración; $U$ es alimento no asimilado por los predadores.

Para cada componente se requiere un estimado de su captura pesquera, biomasa, las razones $\mathrm{P} / \mathrm{B}$ y $\mathrm{Q} / \mathrm{B}, \mathrm{DC} i j, \mathrm{EX} i$, asimilación y EE $i$. Al menos tres de los siguientes parámetros $\mathrm{B}, \mathrm{P} / \mathrm{B}, \mathrm{Q} / \mathrm{B}$ y $\mathrm{EE}$ debe 
ingresarse al modelo. El parámetro desconocido es estimado por el modelo, junto a la respiración, al resolver el sistema de ecuaciones lineales.

En el análisis de las relaciones tróficas y la estructura comunitaria del ecosistema marino de la zona norte se utilizó rutinas de análisis de redes tróficas basadas principalmente en Ulanowicz (1986) y que están incorporadas en el programa Ecopath con Ecosism (EwE). También se determinó propiedades holísticas e índices de flujos del ecosistema basados en conceptos teóricos de Odum (1969) y Ulanowicz (1986). El programa incorpora una rutina propuesta por Ulanowicz (1995) para agregar la trama trófica en niveles tróficos discretos y que permite determinar la distribución de flujos y la eficiencia de transferencia de energía entre niveles tróficos. Además, el programa estimó los siguientes flujos tróficos:

a) la suma de los flujos que se producen en el sistema $\left(\mathrm{F}_{\mathrm{T}}\right)$ que es una medida del tamaño del ecosistema (Jarre-Teichmann, 1998);

b) la producción primaria total que es calculada como la suma de la producciones de todos los grupos que pueden ser atribuibles a la producción primaria (Christensen et al., 2000);

c) la producción primaria requerida para sostener las capturas que es calculada siguiendo la metodología propuesta por Christensen \& Pauly (1993);

d) el índice de reciclaje de Finn (FI), que corresponde a la fracción de $\mathrm{F}_{\mathrm{T}}$ que es dirigida al reciclaje de materia (Finn, 1976 en Christensen et al., 2000);

e) la longitud promedio de las rutas tróficas;

f) el nivel trófico fraccional de cada grupo, lo que permite representar en un gráfico, el diagrama de flujo del modelo ecotrófico balanceado, todos los flujos y biomasas que ocurren en el sistema;

g) el nivel trófico promedio de las pesquerías (Pauly et al., 1998).

Finalmente, se calculó una selección de atributos o indicadores del ecosistema propuestos por Odum (1969) para caracterizar el grado de madurez del ecosistema.

\section{Información de la composición de la dieta y parámetros de entrada para cada grupo funcional}

Las fuentes de los parámetros ingresados al modelo Ecopath para representar la trama trófica del ecosistema pelágico de la zona norte de Chile, se indican en la Tabla 1.
La composición de la dieta se representa como la fracción en peso que cada taxón de presa contribuye a la dieta del predador. La descripción de la composición de la dieta de cada grupo funcional fue tomada de la literatura de grupos de especies del mismo ecosistema como de ecosistemas similares. Las fuentes consultadas se señalan en la Tabla 1 , mientras que la composición de la dieta (DC) de los predadores del sistema se presenta en la Tabla 2.

Debido a la escasa información sobre la alimentación de las aves marinas en la zona norte de Chile, expresada en términos cuantitativos, se tomó como referencia la dieta de aves marinas del ecosistema del sur de Benguela informada por Jarre-Teichmann et al. (1998).

La dieta del lobo marino común se adaptó desde Sielfeld (1997), quien señala a la anchoveta como la presa principal; otras presas son Loligo gahi, Octopus mimus, Isacia conceptionis, Trachinotus paitensis, los que fueron agrupados en importación. La importación al sistema significa el consumo de una presa que no es parte de ese sistema, por lo que deben aparecer constituyendo una parte de la alimentación (Christensen \& Pauly, 1992).

En el grupo funcional tiburones, no existen estudios de alimentación en la zona norte. Por lo tanto, también se consideró como adecuada la información entregada por Jarre-Teichmann et al. (1998) para el mismo grupo en el ecosistema sur de Benguela.

Para la cojinoba del norte sólo se cuenta con el estudio de Wolff \& Aron (1992) de la zona de Coquimbo. Sin embargo, la dieta se encuentra expresada en porcentaje de frecuencias numérica y de ocurrencia, lo que lleva a los autores a caracterizarla como una especie zooplanctófaga. Blaskovic et al. (2002) indican que la dieta de la cojinoba en el área de Callao durante la primavera de 1999 estuvo conformada por peces $(48 \%)$, crustáceos $(50 \%)$ y taliáceos $(0,4 \%)$ en términos de biomasa. El detalle de esto está expresado en términos de logIRI donde los eufáusidos alcanzan el valor más alto. Esta especie no ha sido incluida en ningún otro modelo ecotrófico de sistemas de surgencias, por lo que no ha sido posible contar con información de la dieta expresada como fracción en peso. Por lo tanto se asignó el $100 \%$ al zooplancton.

La composición de la dieta en sardina (Sardinops sagax) se colectó de Oliva et al. (1987). La contribución a la dieta de sardina por taxa de presas fitoplanctónicas es mayor que las presas zooplanctónicas. Sin embargo, esta información se entrega solamente en 
Tabla 1. Fuentes y supuestos de las estimaciones de los parámetros de entrada al modelo Ecopath para cada grupo del modelo ecotrófico del ecosistema pelágico del norte de Chile. B: Biomasa, P/B: producción/biomasa equivalente a la mortalidad total $(P / B=Z=F+M), Q / B$ : Consumo/biomasa, EE: Eficiencia ecotrófica, $Y$ : desembarque anual del grupo.

Table 1. Sources and assumptions for the input parameters in the Ecopath model representing the pelagic ecosystem in northern Chile year 1989. B: biomass, P/B: production/biomass, Q/B: consumption/biomass, EE: ecotrophic efficiency, Y: landing.

\begin{tabular}{|c|c|c|c|c|c|}
\hline Grupo & Dieta & $\begin{array}{c}\text { Biomasa } \\
\left(\text { ton } \cdot k^{-2}\right)\end{array}$ & $\mathbf{P} / \mathbf{B}\left(\mathbf{a n ̃ o} 0^{-1}\right)$ & Q/B $\left(\mathbf{a n ̃ o}{ }^{-1}\right)$ & $\mathbf{E E}$ \\
\hline Aves marinas & $\begin{array}{l}\text { Jarre-Teichmann } \\
\text { et al. }(1998)\end{array}$ & Jarre et al. (1991) & Jarre et al. (1991) & $\begin{array}{l}\text { Jarre et al. } \\
(1991)\end{array}$ & \\
\hline Lobo marino & Sielfeld (1997) & $\begin{array}{l}\text { Neira et al. } \\
(2004)\end{array}$ & Neira et al. (2004) & $\begin{array}{l}\text { Neira et al. } \\
(2004)\end{array}$ & \\
\hline Tiburones & $\begin{array}{l}\text { Jarre-Teichmann } \\
\text { et al. (1998); } \\
\text { Dirección Zonal } \\
\text { de Pesca I y II } \\
\text { Regiones (2001) }\end{array}$ & & $\begin{array}{l}\mathrm{P} / \mathrm{B}=\mathrm{Z} ; \mathrm{Tm}=7 \text { años, edad } \\
\text { de madurez promedio de las } \\
\text { principales especies de la } \\
\text { zona norte; Tm* } \mathrm{M}=1,7 \text { (Frisk } \\
\text { et al. } 2001 \text { ) entonces } \mathrm{M}= \\
0,243 \text { año } \\
\text { Smith, } 1997 \text { ) } \mathrm{Z}=2 \mathrm{M}(\mathrm{Au} \& \\
\end{array}$ & Pauly (1989) & \\
\hline Bonito & $\begin{array}{l}\text { Ojeda \& Jaksic } \\
(1979)\end{array}$ & $\mathrm{B}=\mathrm{Y} / \mathrm{F}$ & Nuñez (1993) & Pauly (1989) & \\
\hline Jurel & $\begin{array}{l}\text { Medina \& } \\
\text { Arancibia } \\
(1990,1992)\end{array}$ & $\mathrm{B}=\mathrm{Y} / \mathrm{F}$ & $\begin{array}{l}\text { M (Serra \& Barría, } 1988 \\
\text { en IFOP, 1991); } F_{\text {Norte }}(\mathrm{L} . \\
\text { Cubillos com.pers) }\end{array}$ & $\begin{array}{l}\text { Medina \& } \\
\text { Arancibia (1995) }\end{array}$ & \\
\hline Caballa & $\begin{array}{l}\text { Medina \& } \\
\text { Arancibia (1990, } \\
\text { 1992) }\end{array}$ & $\mathrm{B}=\mathrm{Y} / \mathrm{F}$ & OLDEPESCA/SELA (1986) & $\begin{array}{l}\text { Ecc. de } \\
\text { Palomares \& } \\
\text { Pauly (1989); } \\
\text { www.fishbase. } \\
\text { org. } \\
\end{array}$ & \\
\hline Cojinoba & $\begin{array}{l}\text { Wolff \& Aron } \\
\text { (1992) Balskovic } \\
\text { et al. }(2002)\end{array}$ & $\mathrm{B}=\mathrm{Y} / \mathrm{F}$ & Medina (2000) & $\begin{array}{l}\text { Ecc. de } \\
\text { Palomares \& } \\
\text { Pauly (1989); } \\
\text { www.fishbase. } \\
\text { org } \\
\end{array}$ & \\
\hline Sardina & $\begin{array}{l}\text { Oliva et } \\
\text { al. (1987); } \\
\text { Armstrong et al. } \\
\text { (1991 en Jarre- } \\
\text { Teichmann et al. } \\
\text { 1998) }\end{array}$ & IFOP (1991) & $\begin{array}{l}\text { F (IFOP, 1991); M (Cubillos, } \\
\text { 1990) }\end{array}$ & $\begin{array}{l}\text { Ecc. de } \\
\text { Palomares \& } \\
\text { Pauly (1989) } \\
\text { utilizando } \\
\text { información } \\
\text { de Optiz (1996 } \\
\text { en García \& } \\
\text { Duarte, 2002) } \\
\end{array}$ & \\
\hline $\begin{array}{l}\text { Peces meso- } \\
\text { pelágicos }\end{array}$ & $\begin{array}{l}\text { Armstrong et } \\
\text { al.(1991 en Jarre- } \\
\text { Teichmann } \text { et } \\
\text { al. 1998); Bleck } \\
(1991)\end{array}$ & & Jarre-Teichmann et al. 1998 & $\begin{array}{l}\text { Jarre-Teichmann } \\
\text { et al. } 1998\end{array}$ & $\begin{array}{l}\text { Jarre- } \\
\text { Teichmann } \\
\text { et al. } \\
(1998)\end{array}$ \\
\hline Anchoveta & $\begin{array}{l}\text { Alamo et al. } \\
(1996)\end{array}$ & $\mathrm{B}=\mathrm{Y} / \mathrm{F}$ & $\begin{array}{l}\text { M (Cubillos, 1991); F (IFOP, } \\
\text { 1991) }\end{array}$ & $\begin{array}{l}\text { Pauly et al. } \\
(1987)\end{array}$ & \\
\hline Zooplancton & $\begin{array}{l}\text { Jarre-Teichmann } \\
\text { et al. }(1998)\end{array}$ & Jarre et al. (1991) & Jarre et al. (1991) & & $\begin{array}{l}\text { Jarre et al. } \\
(1991)\end{array}$ \\
\hline Fitoplancton & & $\begin{array}{l}\text { Neira et al. } \\
(2004)\end{array}$ & Neira et al. (2004) & & $\begin{array}{l}\text { Neira et al. } \\
(2004)\end{array}$ \\
\hline
\end{tabular}


Tabla 2. Matriz de la composición de la dieta de los predadores (expresada como fracción en peso) considerada en el modelo trófico del ecosistema pelágico del norte de Chile, año 1989. Los números representan la fracción en peso del alimento ingerido.

Table 2. Diet composition of the predators (expressed as weight fraction) considered in the Ecopath model representing the pelagic ecosystem model of northern Chile, year 1989.

\begin{tabular}{|c|c|c|c|c|c|c|c|c|c|c|c|}
\hline \multirow[b]{2}{*}{ Presa } & \multicolumn{11}{|c|}{ Depredador } \\
\hline & 1 & 2 & 3 & 4 & 5 & 6 & 7 & 8 & 9 & 10 & 11 \\
\hline 1. Aves marinas & & & & & & & & & & & \\
\hline 2. Lobo marino & & & 0,002 & & & & & & & & \\
\hline 3. Tiburones & & & 0,05 & & & & & & & & \\
\hline 4. Bonito & 0,001 & & 0,025 & & & & & & & & \\
\hline 5. Jurel & 0,008 & & 0,012 & & & & & & & & \\
\hline 6. Caballa & 0,001 & & 0,178 & & & & & & & & \\
\hline 7. Cojinoba & 0,001 & & 0,025 & & & & & & & & \\
\hline 8. Sardina & 0,084 & & 0,012 & & & & & & & & \\
\hline 9. Peces mesopelágicos & 0,103 & & & & 0,480 & 0,350 & & & & & \\
\hline 10. Anchoveta & 0,365 & 0,77 & 0,09 & 0,950 & 0,060 & 0,320 & & & & & \\
\hline 11. Zooplancton & 0,105 & & & 0,050 & 0,450 & 0,300 & 1,000 & 0,260 & 1,000 & 0,850 & 0,220 \\
\hline 12. Fitoplancton & & & & & & & & 0,740 & & 0,150 & 0,410 \\
\hline 13. Detritos & & & & & & & & & & & 0,370 \\
\hline Importación & 0,333 & 0,23 & 0,598 & & 0,010 & 0,030 & & & & & \\
\hline
\end{tabular}

un gráfico de la composición volumétrica porcentual mensual de fitoplancton y zooplancton en los contenidos estomacales, siendo imposible contar con los datos brutos en la matriz de DC. Armstrong et al. (1991 en Jarre-Teichmann et al., 1998) indican para Sardinops sagax del sur de Benguela un 33\% de zooplancton y un $67 \%$ de fitoplancton por lo que los valores considerados en la matriz se consideraron aceptables.

Siguiendo a Amstrong et al. (1991 en JarreTeichmann et al., 1998), se consideró que la dieta de peces mesopelágicos corresponde a $100 \%$ de zooplancton, ya que en la sección sur del ecosistema de Benguela, estos peces se alimentan de un $40 \%$ de copépodos y de un $60 \%$ de eufáusidos. El único estudio de la alimentación de peces mesopelágicos en el norte de Chile es el de Bleck (1991), quien describe la alimentación de cuatro especies mesopelágicas y señala que los copépodos y eufáusidos son los grupos más importantes en la dieta, pero todo ello expresado sólo en términos de ocurrencia y contribución numérica.

En el caso de la anchoveta (Engraulis ringens), se consideró la información de Alamo et al. (1996), quienes indican que la composición trófica de la anchoveta del Perú en términos de biomasa ingerida corresponde un $85 \%$ a zooplancton y $15 \%$ a fitoplancton.
Para un ecosistema explotado, el modelo requiere de las capturas pesqueras, la cual es equivalente a exportaciones desde el área y por lo tanto son valores de entrada en el modelo. Se consideró que las capturas son iguales a los desembarques oficiales, los que se obtuvieron desde el Anuario Estadístico de Pesca del Servicio Nacional de Pesca (SERNAPESCA, 1989).

Las biomasas y desembarques se ingresaron divididas por el área de estudio (ton $\left.\cdot \mathrm{km}^{-2} \cdot \mathrm{anno}^{-1}\right)($ Tabla 3 ). En este estudio se supuso que la tasa de migración neta es igual a cero y que las únicas exportaciones desde el sistema son bajo la forma de las capturas pesqueras.

\section{Balance del modelo}

Una vez completa la matriz de los parámetros de entrada básicos y la matriz de la composición de la dieta, se ejecutó el programa Ecopath con Ecosim (EwE). Para verificar el balance de masa de cada grupo funcional, se revisó los siguientes parámetros de salida indicados por el programa:

- Eficiencia Ecotrófica $\left(\mathrm{EE}_{i}\right)$ de cada grupo, en que los valores deben estar entre 0 y 1 . Valores $>1$ son inconsistentes ya que no es posible que se consuma o capture más biomasa que la producida por el grupo. 
- Eficiencia bruta de conversión de alimento $\left(\mathrm{GE}_{i}\right)$ que se define como la razón entre la producción y el consumo y en la mayoría de los casos fluctúa entre 0,05 y 0,3 .

- Respiración/Asimilación (R/A) no puede ser $>1$, debido a que lo que se respira no puede exceder lo asimilado.

Para aquellos valores inconsistentes se hicieron cambios en los datos de entrada ya sea de $\mathrm{B}_{i}, \mathrm{P}_{i} / \mathrm{B}_{i}$, o $\mathrm{DC}_{i j}$ siguiendo los criterios presentados en Christensen et al. (2000) hasta obtener valores aceptables en los parámetros de salida (i.e. EE, GE, R/A).

\section{RESULTADOS}

En la Tabla 2 se entrega la composición de la dieta de los predadores del modelo final balanceado y en la Tabla 3 los valores de los parámetros de entrada y los calculados por el programa para el balance final del modelo del ecosistema pelágico de la zona norte de Chile durante 1989.

\section{Características generales del ecosistema pelágico del norte de Chile}

Los flujos totales (FT) que son una medida del tamaño del sistema en términos de flujos, se estimaron en 38.674 ton $\cdot \mathrm{km}^{-2} \cdot$ año $^{-1}$, que corresponden a la suma de todos los flujos (consumo, exportaciones, respiración y flujos hacia el detritos) dentro del sistema (Tabla 4). Los mayores flujos corresponden al consumo con un $34 \%$, secundariamente los flujos hacia el detrito $(31,2 \%)$ y exportación $(24 \%)$, y en menor porcentaje a la respiración (11\%).

La producción primaria del sistema calculada por Ecopath alcanzó $13.452,84$ ton $\cdot \mathrm{km}^{-2} \cdot \mathrm{año}^{-1}$. La producción primaria requerida para sustentar las capturas totales de 91,01 ton $\cdot \mathrm{km}^{-2} \cdot \mathrm{anno}^{-1}$ fue estimada por Ecopath en $1.321,37$ ton $\cdot \mathrm{km}^{-2}$. año ${ }^{-1}$ correspondiendo a un $6,7 \%$ de la producción total del sistema.

El nivel trófico (NT) promedio de la pesquería del norte de Chile fue de 2,7 lo que refleja que las capturas en 1989 se sostuvieron en especies ubicadas en los niveles inferiores de la trama trófica, principalmente en sardina y secundariamente en anchoveta.

Tabla 3. Valores de los parámetros de entrada y estimados (en negritas) por el programa Ecopath para el balance final del modelo que representa el ecosistema pelágico del norte de Chile. Símbolos: NT: nivel trófico; P/B: producción/biomasa; $Q / B$ : consumo/biomasa; EE: eficiencia ecotrófica; GE: eficiencia bruta de conversión de alimento; $R / A$ : razón respiración/asimilación.

Table 3. Input and estimated parameters (bold) obtained from Ecopath representing the final balanced model representing the pelagic ecosystem in the northern Chile year 1989. Symbols: NT: trophic level, P/B: production/ biomass, Q/B: consumption/biomass, EE: ecotrophic efficiency GE: gross efficiency; R/A: respiration per unit off biomass.

\begin{tabular}{|c|c|c|c|c|c|c|c|c|}
\hline \multirow[b]{2}{*}{ Grupo } & \multicolumn{8}{|c|}{ Parámetros } \\
\hline & NT & $\begin{array}{c}\text { Desembarques } \\
\text { ton } / \mathbf{k m}^{\mathbf{2}}\end{array}$ & $\begin{array}{l}\text { Biomasa } \\
\text { ton } / \mathbf{k m}^{2}\end{array}$ & $\begin{array}{c}\mathbf{P} / \mathbf{B} \\
\mathbf{a n n o}^{-1}\end{array}$ & $\begin{array}{c}\mathbf{Q} / \mathbf{B} \\
\mathbf{a n ̃ o}^{-1}\end{array}$ & $\mathbf{E E}$ & GE & $\mathbf{R} / \mathbf{A}$ \\
\hline 1. Aves marinas & 3,8 & & 0,280 & 0,040 & 19,200 & 0,000 & 0,002 & 0,997 \\
\hline 2. Lobo marino & 4,0 & 0,005 & 0,030 & 1,050 & 15,000 & 0,174 & $\mathbf{0 , 0 7 0}$ & 0,913 \\
\hline 3. Tiburones & 4,4 & 0,003 & 0,007 & 0,486 & 9,640 & 0,960 & 0,050 & 0,937 \\
\hline 4. Bonito & 3,8 & 0,009 & 0,019 & 0,987 & 29,450 & 0,856 & 0,034 & 0,958 \\
\hline 5. Jurel & 3,7 & 9,658 & 91,805 & 0,355 & 8,110 & 0,303 & 0,044 & 0,945 \\
\hline 6. Caballa & 3,8 & 1,078 & 1,331 & 1,200 & 10,279 & 0,686 & 0,117 & $\mathbf{0 , 8 5 4}$ \\
\hline 7. Cojinoba & 3,1 & 0,024 & 0,027 & 1,189 & 3,700 & 0,885 & 0,321 & 0,598 \\
\hline 8. Sardina & 2,3 & 42,063 & 44,211 & 1,461 & 8,678 & 0,658 & 0,168 & 0,790 \\
\hline 9. Peces mesopelágicos & 3,1 & & 323,470 & 1,200 & 12,000 & 0,940 & 0,100 & 0,875 \\
\hline 10. Anchoveta & 2,9 & 38,169 & 47,065 & 2,011 & 12,050 & 0,954 & 0,167 & 0,791 \\
\hline 11. Zooplancton & 2,1 & & 87,318 & 64,680 & 85,831 & 0,987 & 0,754 & 0,058 \\
\hline 12. Fitoplancton & 1,0 & & 112,107 & 120,000 & & 0,309 & & \\
\hline 13. Detrito & 1,0 & & 1,000 & & & 0,238 & & \\
\hline
\end{tabular}


Tabla 4. Índices globales calculados por Ecopath para el modelo que representa el ecosistema pelágico del norte de Chile para el año 1989.

Table 4. Global indices calculated by Ecopath for the model representing the pelagic ecosystem of northern Chile, year 1989.

\begin{tabular}{|c|c|}
\hline Indicador & Valor \\
\hline Suma de todo el consumo (ton $\cdot \mathrm{km}^{-2} \cdot \mathrm{anno}^{-1}$ ) & $13.091,81$ \\
\hline Suma de todas las exportaciones (ton $\cdot \mathrm{km}^{-2} \cdot \mathrm{anno}^{-1}$ ) & $9.278,51$ \\
\hline Suma de todos los flujos de respiración (ton $\cdot \mathrm{km}^{-2} \cdot \mathrm{año}^{-1}$ ) & $4.244,01$ \\
\hline Suma de todos los flujos al detrito $\left(\right.$ ton $\left.\cdot \mathrm{km}^{-2} \cdot \mathrm{año}^{-1}\right)$ & $12.060,16$ \\
\hline Flujos totales del sistema (ton $\cdot \mathrm{km}^{-2} \cdot \mathrm{año}^{-1}$ ) & 38.674 \\
\hline Suma de toda la producción (ton $\cdot \mathrm{km}^{-2} \cdot \mathrm{año}^{-1}$ ) & 19.684 \\
\hline Nivel trófico promedio de la pesquería & 2,7 \\
\hline Eficiencia bruta (capturas/producción primaria neta) & 0,006765 \\
\hline Producción primaria neta total calculada (ton $\cdot \mathrm{km}^{-2} \cdot \mathrm{año}^{-1}$ ) & $13.452,84$ \\
\hline Producción primaria total/respiración total $\left(\mathrm{año}^{-1}\right)$ & 3,17 \\
\hline Producción primaria total/biomasa total $\left(\mathrm{año}^{-1}\right)$ & 19,01 \\
\hline Producción primaria requerida para sustentar las capturas $(\%)$ & 6,7 \\
\hline Eficiencia media de transferencia trófica (\%) & 9,8 \\
\hline Biomasa total (excluyendo el detrito) $\left(\right.$ ton $\cdot \mathrm{km}^{-2}$ ) & 707,673 \\
\hline Índice de reciclaje de Finn & 2,79 \\
\hline Longitud promedio de las rutas tróficas & 2,34 \\
\hline Capturas totales $\left(\right.$ ton $\cdot \mathrm{km}^{-2} \cdot \mathrm{año}^{-1}$ ) & 91,01 \\
\hline
\end{tabular}

Otros índices globales que describen el ecosistema pelágico que dan cuenta de las características del ecosistema en términos de flujos y de madurez se entregan en la Tabla 4, los que serán discutidos posteriormente.

\section{Diagrama de flujos tróficos}

Los niveles tróficos fueron estimados por el programa a partir del promedio ponderado de los niveles tróficos de la presa y se distribuyeron desde 1,0 para los productores primarios (fitoplancton) y detrito hasta 4,4 para los depredadores tope como el grupo tiburones (Fig. 2). Los grupos zooplancton (NT = $2,1)$ y sardina $(\mathrm{NT}=2,3)$ se ubicaron alrededor del segundo nivel trófico debido a su alta depredación sobre el primer nivel trófico. En seguida se ubicaron los peces mesopelágicos, anchoveta y cojinoba, altamente zooplanctófagos. El resto de los grupos se ubicó en niveles cercanos y superiores a NT $=4$ debido a que son principalmente carnívoros.
Los principales flujos en el ecosistema, medidos como flujos de consumo, se producen entre los consumidores primarios (zooplancton) $(\mathrm{Q}=$ 7.494 ton $\mathrm{km}^{-2}$ año ${ }^{-1}$ ) y los productores primarios $\mathrm{y}$ desde zooplancton hacia peces mesopelágicos $\left(\mathrm{Q}=3.881\right.$ ton $\left.\cdot \mathrm{km}^{-2} \cdot \mathrm{año}^{-1}\right)$. Se destacan también otros flujos dentro del ambiente pelágico que van desde productores primarios y zooplancton hacia sardina $\left(\mathrm{Q}=383,7\right.$ ton $\left.\cdot \mathrm{km}^{-2} \cdot \mathrm{anno}^{-1}\right)$ y anchoveta $(\mathrm{Q}$ $=567,1$ ton $\left.\cdot \mathrm{km}^{-2} \cdot \mathrm{anno}^{-1}\right) ; \mathrm{y}$ desde zooplancton, peces mesopelágicos y anchoveta hacia jurel $(\mathrm{Q}=744,5$ ton $\cdot \mathrm{km}^{-2} \cdot \mathrm{anno}^{-1}$ ) (Fig. 2).

\section{Eficiencia de transferencia de energía}

La eficiencia media de transferencia fue estimada en 9,8\% (Fig. 3). La eficiencia de transferencia entre niveles tróficos discretos expresa cuán óptimo es el transporte de energía de un nivel trófico al siguiente, observándose que es mayor desde los niveles tróficos primarios (I a II) y disminuye hacia los niveles tróficos mayores. 


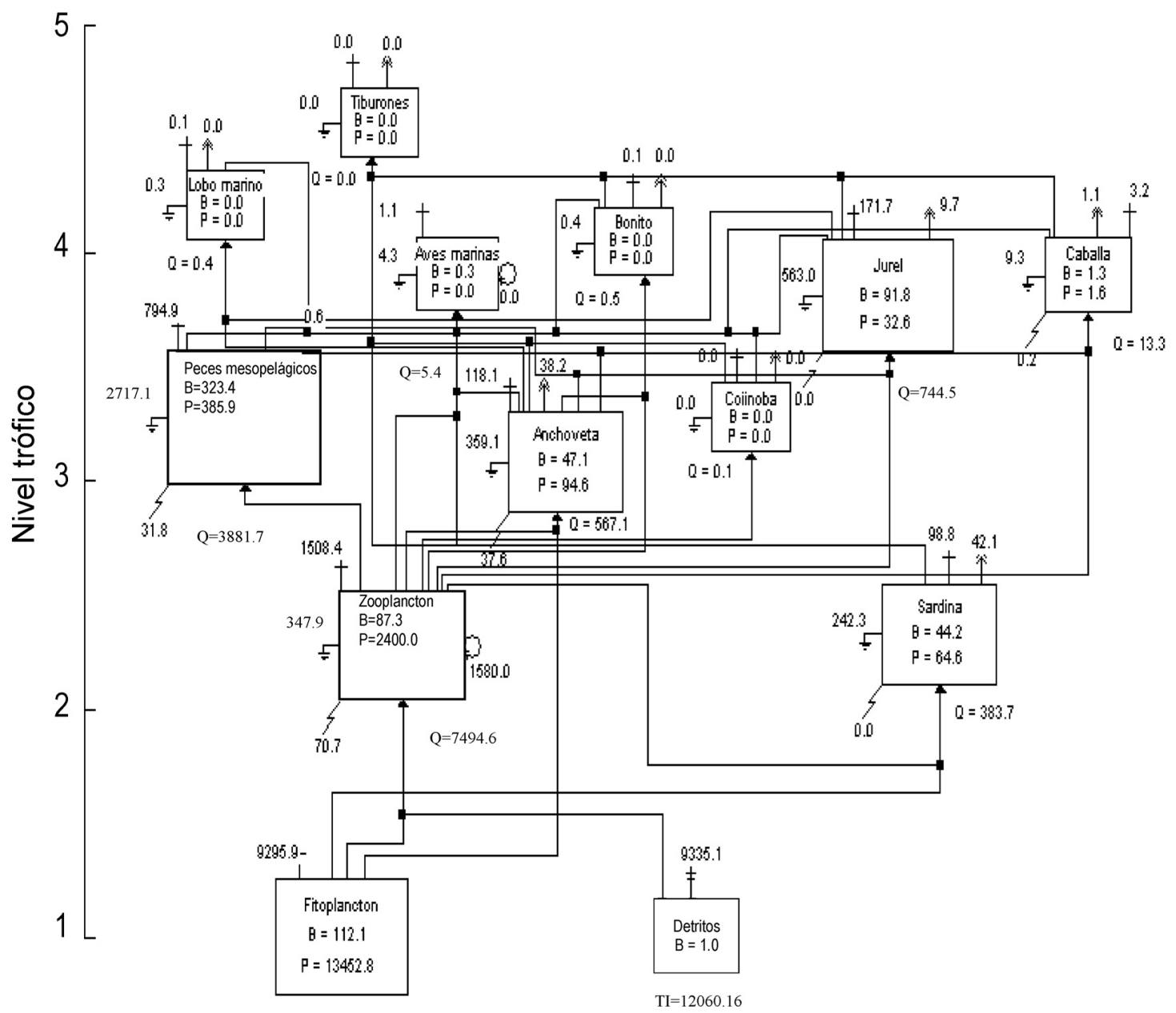

Figura 2. Diagrama de flujos que representan el ecosistema pelágico de la zona norte de Chile en el año 1989. El tamaño de las cajas es proporcional a la raíz cuadrada de la biomasa de los componentes. Las cajas se distribuyen en el eje vertical de acuerdo a su nivel trófico. Los flujos están expresados en ton $\cdot \mathrm{km}^{-2} \cdot \mathrm{anno}^{-1}$. P: producción; B: biomasa; Q: consumo; TI: Suma de todos los flujos al detrito.

Figure 2. Flow diagram representing the pelagic ecosystem in northern Chile in year 1989. The box size is proportional to the square root of biomass of each compartment. Boxes are aligned in the "Y" axe according to their trophic level. Flows are expressed in ton $\cdot \mathrm{km}^{-2} \cdot \mathrm{year}^{-1}$. P: production; B: biomass; Q: consumption; TI: sum of all flows into detritus. 


\section{Eficiencia media de transferencia: 9,8 \%}

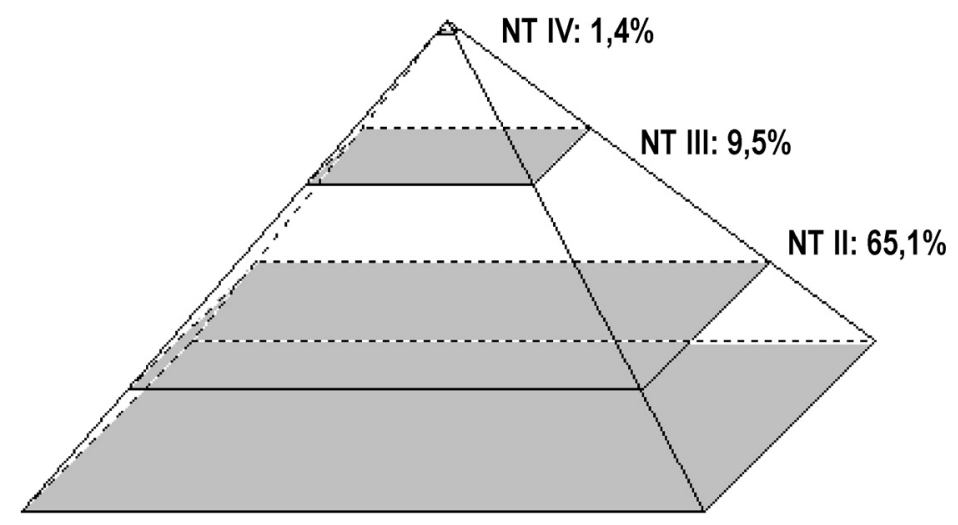

Figura 3. Pirámide trófica de los flujos del ecosistema pelágico de la zona norte de Chile, año 1989. El volumen de cada nivel trófico (NT) discreto es proporcional a las transferencias totales en este nivel. El compartimiento de la base representa los flujos totales al nivel trófico discreto II (herbívoros).

Figure 3. Trophic pyramid of the pelagic ecosystem in northern Chile, year 1989. The volume of each discrete trophic level (NT) is proportional to the throughput (total flow) at that level; the bottom compartment represents the discrete trophic level II composed by herbivores (trophic level II).

\section{DISCUSIÓN}

La falta de información referente a los principales grupos funcionales del ecosistema en estudio es una realidad preocupante. Sin embargo, esta limitación no puede ser esgrimida para no avanzar en descripciones cualitativas y semi-cuantitativas de su trama trófica. En este sentido, se utilizaron como parámetros de entrada, en algunos grupos, valores encontrados en la literatura especializada que se consideraron aceptables, ya que corresponden a estimaciones realizadas para grupos funcionales similares en ecosistemas comparables. Por lo tanto, muchos de los resultados deben ser analizados considerando la incertidumbre en la data y deberán ser confirmados o mejorados a medida que más información y de mejor calidad sea generada en el ecosistema pelágico de la zona norte de Chile.

Considerando lo anterior, el modelo construido en este estudio requirió de ajustes a la matriz de la dieta y de otros parámetros de entrada para llegar al balance final. Cambios en la matriz de la dieta fueron necesarios considerando que la composición de la dieta para algunos grupos se obtuvo a partir de información trófica de especies similares en ecosistemas comparables, como por ejemplo en el caso de aves marinas, tiburones y peces mesopelágicos.
Uno de los resultados de la modelación de una trama trófica es la identificación de las áreas deficitarias del conocimiento científico respecto del ecosistema que se estudia. Como era de esperar, los datos de entrada más confiables corresponden a los parámetros de entrada de los recursos pesqueros. Sin embargo, este estudio revela un desconocimiento prácticamente absoluto de los parámetros poblacionales y ecológicos en grupos pertenecientes tanto a los niveles tróficos superiores como inferiores de la trama trófica. Por lo tanto, las necesidades de investigación deben orientarse a completar y, sobre todo, a actualizar la información básica de los principales grupos recursos pesqueros del ecosistema pelágico del norte de Chile.

Al comparar los flujos totales $\left(\mathrm{F}_{\mathrm{T}}=38.674\right.$ ton $\cdot \mathrm{km}^{-2} \cdot \mathrm{anno}^{-1}$ ) de este sistema con otros sistemas de surgencia, se tiene que este sistema es de mayor tamaño (en términos de flujos sustentados) que el sistema de Perú modelado en tres periodos con valores de 29.600 ton $\cdot \mathrm{km}^{-2} \cdot$ año $^{-1}$ (años 1953-1959), 29.382 ton $\cdot \mathrm{km}^{-2} \cdot \mathrm{año}^{-1}(1963-1969)$ y 33.539 ton $\cdot \mathrm{km}^{-2} \cdot \mathrm{año}^{-1}$ (1973-1979), con excepción del periodo 1964-1971 que alcanzó un valor sobre 60.000 ton $\cdot \mathrm{km}^{-2} \cdot$ año $^{-1}$ antes del colapso de la pesquería de la anchoveta (Jarre-Teichmann et al., 1998). En relación con los valores obtenidos por Neira et al. (2004) para el eco- 
sistema de Chile central para el año 1992 y 1998, el ecosistema del norte de Chile habría sido de mayor tamaño que el determinado en 1992, cuyo valor de flujos totales fue de $30.619,737 \mathrm{ton} \cdot \mathrm{km}^{-2}$. año $0^{-1} \mathrm{y}$ menor que el sistema de 1998 con un valor de 43.964,201 ton $\cdot \mathrm{km}^{-2} \cdot \mathrm{año}^{-1}$.

La producción primaria del sistema calculada por Ecopath alcanzó $13.452,84$ ton $\cdot \mathrm{km}^{-2} \cdot \mathrm{año}^{-1}$, valor que se encuentra en el rango del ecosistema sur de Benguela durante el periodo de 1980-89 (aproximadamente 12.000 ton $\cdot \mathrm{km}^{-2} \cdot \mathrm{anno}^{-1}$ ) y del ecosistema de Perú durante el periodo 1972-81 (aproximadamente 17.000 ton $\cdot \mathrm{km}^{-2} \cdot \mathrm{año}^{-1}$ ) (Jarre-Teichmann et al., 1998).

La razón producción primaria total/respiración total $(\mathrm{PP} / \mathrm{R})$ calculada para el norte de Chile es de 3,17 . Odum (1971) considera esta razón importante en la descripción de la madurez de un ecosistema. $\mathrm{PP} / \mathrm{R}$ es $>1$ al principio del desarrollo del sistema, ya que la producción excede a la respiración. La $\mathrm{PP} / \mathrm{R}$ calculada para este ecosistema excede el límite de 1 para sistemas maduros, por lo tanto este valor corresponde a un sistema alejado de la madurez o inmaduro (Christensen et al., 2000). Otro parámetro que describe la madurez del sistema es el índice de reciclaje de Finn, en que sistemas maduros se caracterizan por un mayor reciclaje de materia y por rutas o cadenas tróficas largas (Odum, 1969). El valor obtenido en este estudio ( $\mathrm{FI}=2,79 \%)$ es bajo comparado con valores señalados por Baird \& Ulanowicz (1993) para cuatro sistemas estuarinos y por Wolf (1994) para la bahía de Tongoy. Sin embargo, resulta comparable a los valores calculados para el ecosistema de Chile central (Arancibia et al., 2003), lo que también indica que es un sistema alejado de la madurez, lo que parece ser una generalidad en ecosistemas de surgencia.

La eficiencia media de transferencia de energía se estimó en 9,8\%, valor similar al mínimo del rango (10\%-20\%) comúnmente indicado para ecosistemas marinos (Christensen \& Pauly, 1993). Sin embargo, este valor está dentro de la regla de que los sistemas de surgencia son altamente ineficientes, con una eficiencia de transferencia promedio global cercana a 10\% (Pauly \& Christensen, 1995; Jarre Teichmann et al., 1998).

La producción primaria requerida para sustentar las capturas totales del sistema fue de un $6,7 \%$ de la producción total del sistema. Comparaciones de PPR realizadas por Jarre-Teichmann et al. (1998) entre otros ecosistemas de surgencia como Perú, África del
Sur, Namibia y California indican que PP/R puede variar entre un $4 \%$ y $15 \%$ de la producción total, de tal manera que el valor estimado para el norte de Chile está dentro del rango característico de ecosistemas marinos similares.

El análisis general del diagrama de flujo indica que los principales flujos en el ecosistema, medidos como flujos de consumo, se producen entre los consumidores primarios (zooplancton) y los productores primarios, y desde zooplancton hacia peces mesopelágicos. El zooplancton que es un grupo que presenta una alta biomasa y que se alimenta principalmente de fitoplancton, fue situado en un bajo nivel trófico al igual que la sardina. Este grupo es una presa importante para la mayoría de los grupos del ecosistema modelado. El grupo peces mesopelágicos fue asignado por sobre el tercer nivel trófico estimando el programa una alta biomasa que es predada principalmente por jurel y caballa.

El nivel trófico promedio de la pesquería del norte de Chile $(\mathrm{NT}=2,7)$ indica que las capturas en 1989 se sostuvieron en especies ubicadas en los niveles inferiores de la trama trófica, principalmente en sardina y secundariamente en anchoveta, contrariamente a lo ocurrido en el ecosistema de Chile central en que las capturas durante 1992 y 1998 estuvieron sustentadas por niveles tróficos mayores a 3 correspondiendo principalmente a jurel $(\mathrm{NT}=3,7)$ (Neira et al., 2004; Arancibia \& Neira, 2005).

La eficiencia bruta de la pesquería (captura/producción primaria) fue calculada en $0,6 \%$. Valores cercanos a 1 son típicos de sistemas con pesquerías que capturan peces de nivel trófico bajo (pesquerías en zonas de surgencia), mientras que los valores bajos son característicos de sistemas en que los stocks de peces se encuentran subexplotados o donde la pesquería se concentra sobre los predadores tope (Christensen \& Pauly, 1992). El valor 0,6\% de la eficiencia bruta de la pesquería en 1989 revela que ésta se sostuvo en peces de bajo nivel trófico. Sardina española y anchoveta fueron los recursos pesqueros más importantes durante 1989, con desembarques de 42,1 y 38,2 ton $\cdot \mathrm{km}^{-2}$ por año respectivamente.

Un análisis de la composición específica de los recursos capturados desde 1984 indica un claro predominio de la sardina $(80 \%)$, seguido por el jurel $(15 \%)$ y una incidencia errática de la anchoveta, especie que alcanzó en 1986 la cifra histórica más alta, contribuyendo con el 33\% de las capturas. Este recurso se dice que habría colapsado en 1987 debido a la alta tasa de explotación a que fue sometido en 
1986 y a la presencia de El Niño 1987, pero mostró un marcado repunte en 1988. En 1988 los estudios de evaluación de stock ya señalaban que el stock de sardina entraba en una etapa de colapso y que la anchoveta comenzaba a presentar una rápida recuperación frente a condiciones ambientales favorables (Martínez et al., 1989).

En general, los resultados de este estudio indican que el ecosistema pelágico de la zona norte de Chile, al igual que el ecosistema de Chile central (Aranciba et al., 2003), se puede caracterizar como un sistema que está alejado de la madurez, baja eficiencia en la transferencia de energía, cadenas tróficas cortas y un bajo reciclaje de materia, lo que es consistente con lo informado para los principales ecosistemas de surgencia del mundo (Jarre-Teichmann \& Christensen, 1998).

El modelo obtenido con Ecopath para el ecosistema pelágico del norte de Chile permite tener una idea aproximada del ecosistema en función de los principales flujos, como la respiración, consumo, producción total entre otra serie de índices ecológicos. Pero para ir hacia un manejo multiespecífico de pesquerías de la zona norte es necesario en una primera etapa, incluir más grupos en el modelo para obtener una mejor representación de la trama trófica, realizar nuevos estudios tendientes a actualizar y/o generar nueva información relativa a parámetros de entrada proveniente de los grupos funcionales del mismo ecosistema, incentivar a los autores a publicar en revistas especializadas sus trabajos ya que existe mucha literatura gris sobre alimentación y otros parámetros biológico en peces, lo que permitirá a futuro lograr un modelo del ecosistema pelágico del norte Chile de mejor calidad y mayor robustez cuantitativa.

\section{AGRADECIMIENTOS}

Los autores agradecen al Fondo de Investigación Pesquera por el financiamiento al Proyecto FIP №2001-29 "Enfoque metodológico para el análisis ecosistémico en la administración de pesquerías de la Zona Central de Chile", que permitió el desarrollo de esta investigación. A los revisores anónimos por sus comentarios valiosos que permitieron mejorar el manuscrito final. Sergio Neira agradece a Institute de Recherche pur le Développement (IRD, Francia) y a Marine Biology Research Institute (University of Cape Town, South Africa) por su gentileza al financiar sus estudios de doctorado.

\section{REFERENCIAS}

Alamo, A., I. Navarro, P. Espinoza \& P. Zubiate. 1996. Relaciones tróficas, espectro alimentario y ración de alimentación de las principales especies pelágicas en el verano de 1996. Inf. Inst. Mar. Callao, Perú, 122: 36-46.

Allen, K.R. 1971. Relation between production and biomass. J. Fish. Res. Bd. Canada, 28: 1573-1581.

Arancibia, H., S. Neira, V. Christensen, R. Olson, F. Arreguín-Sánchez, L. Cubillos, R. Quiñones, C. Gatica \& M. Medina. 2003. Enfoque metodológico para el análisis ecosistémico en la administración de pesquerías de la zona central de Chile. Informe Final Proyecto FIP N ${ }^{\circ}$ 2001-29: 278 pp.

Arancibia, H. \& S. Neira. 2005. Long-term changes in the mean trophic level of Central Chile fishery landings. Sci. Mar., 69(2): 295-300.

Au, D.W. \& S.E. Smith. 1997. A demographic method with population density compensation for estimating productivity and yield per recruit of the leopard shark (Triakis semifasciata). Can. J. Fish. Aquat. Sci., 54: 415-420.

Baird, D. \& R. Ulanowicz. 1993. Comparative study on the trophic structure, cycling and ecosystem properties of four tidal estuaries. Mar. Ecol. Prog. Ser., 99: 221-237.

Bernal, P. 1990. La oceanografía del sistema de corrientes de Chile-Perú en relación a las pesquerías pelágicas: una revisión. En: M.A. Barbieri (ed.). Perspectivas de la actividad pesquera en Chile. Escuela de Ciencias del Mar, UCV, Valparaíso, pp. $35-48$.

Blaskovic, V., P. Espinoza, F. Torriani \& I. Navarro. 2002. Hábitos alimentarios y variaciones de la dieta de los principales peces pelágicos y demersales en la primavera de 1999. Dirección de Investigación en Biodiversidad Ecología Trófica y Reproducción. (http://www.imarpe.gob.pe/imarpe/pelagicos.php).

Bleck, J.Z. 1991. Descripción de los hábitos alimentarios de cuatro especies de peces mesopelágicos del norte de Chile, Arica-río Loa $\left(18^{\circ} 25^{\prime} \mathrm{S}-72^{\circ} 04^{\prime} \mathrm{W}\right.$ a $\left.19^{\circ} 09^{\prime} \mathrm{S}-70^{\circ} 36^{\prime} \mathrm{W}\right)$ : Triphoturus oculeus, Loweina sp. (Myctophidae), Vinciguerria sp. (Photichthydae) y Cyclothone sp. (Gonostomatidae). Informe Práctica Profesional, Biología Marina. Universidad Arturo Prat, 51 pp.

Christensen, V. \& D. Pauly. 1992. A guide to Ecopath II program (version 2.1) ICLARM Software, 6: $72 \mathrm{pp.}$

Christensen, V. \& D. Pauly. 1993. (eds.). Trophic 
models of acuatic ecosystems, ICLARM Conf. Prod. 26. Manila: ICLARM, $390 \mathrm{pp}$.

Christensen, V., C.J. Walters \& D. Pauly. 2000. Ecopath with Ecosim: A user's guide. Fisheries Centre, University of British Columbia Vancouver, Canada and ICLARM, Penang, Malaysia, 131 pp.

Cubillos, L. 1990. Proyecciones del estado futuro de la biomasa del stock de sardina (Sardinops sagax) por aproximación global. Programa INPESCON 1990. Universidad Arturo Prat, Departamento de Ciencias del Mar. Iquique-Chile. Doc. Téc., 44 A(5): 25 pp.

Cubillos, L. 1991. Estimación mensual de la biomasa, reclutamiento y mortalidad por pesca de la anchoveta (Engraulis ringens) de la zona norte de Chile en el periodo 1986-1989. Biol. Pesq., 20: 49-59.

Dirección Zonal de Pesca I-II Regiones. 2001. Antecedentes biológicos-pesqueros y propuestas de ordenación para el recurso Tiburón. Informe Técnico COZOPE I-II N 02/2001, 20 pp.

Escobar, J.J.R. 2001. El aporte del enfoque ecosistémico a la sostenibilidad pesquera. CEPAL-SERIE Recursos naturales e infraestructura $\mathrm{N}^{\circ} 39$. Publicación de las Naciones Unidas, 55 pp.

Frisk, M.G., T.J. Miller \& M.J. Fogarty. 2001. Estimation and analysis of biological parameters in elasmobranch fishes: a comparative life history study. Can. J. Fish. Aquatic. Sci., 58: 969-981.

Fuenzalida, R. 1992. Anomalías oceanográficas y meteorológicas durante el desarrollo de El Niño 1991-1992 en la zona de Iquique (20¹8’S). Inv. Pesq. (Chile), 37: 67-72.

Instituto de Fomento Pesquero (IFOP). 1991. Diagnóstico de las principales pesquerías nacionales 1990. Pesquerías pelágicas zona norte (I-IV Región). Convenio CORFO-IFOP. SGI-IFOP 91/8: $94 \mathrm{pp}$.

García, C.B. \& L.O. Duarte. 2002. Consumption to biomass $(\mathrm{Q} / \mathrm{B})$ ratio and estimates of $\mathrm{Q} / \mathrm{B}$-predictor parameters for Caribbean fishes. Naga, The ICLARM Quarterly, 25(2): 19-31.

Jarre, A., P. Muck \& D. Pauly. 1991. Two approaches for modelling fish stock interactions in the peruvian upwelling ecosystem. ICES Mar. Sci. Symp., 193: 171-184.

Jarre-Teichmann, A. \& V. Christensen. 1998. Comparative modellling of trophic flows in four large upwelling ecosystems: global versus local effects. En: M. Durand, P. Cury, P. Mendelson, C. Roy, A. Bakun \& D. Pauly (eds.). Global versus local changes in upwelling ecosystems, Paris, ORSTOM, pp. 424-443.
Jarre-Teichmann, A., L.J. Shannon, C.L. Moloney \& P.A. Wickens. 1998. Comparing trophic flows in the southern Benguela to those in other upwelling ecosystems. S.C. Pillar, C.L. Moloney, A.I.L. Payne \& F.A. Shillington (eds.). S. Afr. J. Mar. Sci., 19: 391-414.

Jarre-Teichmann, A. 1998. The potencial rol of mass balance models for the management of upwelling ecosystems. Ecol. Applic., 8(1): 93-103.

Mackinson, S., M. Vasconcellos, T. Pitcher \& C. Walters. 1997. Ecosystem impacts of the harvesting small pelagic fish in upwelling systems: using a dynamic mass balance model. Forage fishes in marine ecosystems. Alaska Sea grant College Program, AKSG-97-01: 731-749.

Martínez, C., G. Bohm \& L. Caballero. 1989. La pesquería pelágica de la zona norte de Chile y su relación con las condiciones ambientales. CPPS, Boletín ERFEN, 29: 31-36.

Medina, M. \& H. Arancibia. 1990. Interacciones tróficas entre el jurel (Trachurus murphyi) y la caballa (Scomber japonicus) en el ecosistema pelágico de la zona de Iquique. Programa INPESCON 1989. Universidad Arturo Prat, Departamento de Ciencias del Mar, Iquique, Doc. Téc., 44B(10): 55 pp.

Medina, M. \& H. Arancibia. 1992. Interacciones tróficas entre el jurel (Trachurus murphyi) y la caballa (Scomber japonicus) en el ecosistema pelágico de la zona norte. Invest. Cient. y Tec., Serie: Ciencias del Mar, 2: 67-78.

Medina, M. \& H. Arancibia. 1995. Relaciones tróficas del jurel en la zona norte (Regiones I y II). En: Estudio biológico pesquero sobre el recurso jurel en la zona norte (Regiones I y II). Instituto de Fomento Pesquero/Universidad Arturo Prat/Instituto de Investigación Pesquera, Informe Final Proyecto FIP 93-17: 221 pp.

Medina, M. 2000. Propuesta de medidas de administración para la explotación del recurso cojinoba del norte (Seriolella violacea) de la I y II Regiones. Seminario. Programa de Magíster en Ciencias Mención Pesquerías, Universidad de Concepción, Concepción, 25 pp.

Neira, S., A. Arancibia \& L. Cubillos. 2004. Comparative analysis of trophic structure of commercial fishery species off Central Chile in 1992 and 1998. Ecol. Model., 172: 233-248.

Núñez, E. 1993. Crecimiento, mortalidad y rendimiento potencial del bonito (Sarda chilensis $\mathrm{Cu}-$ vier, 1831) en el norte de Chile. Tesis de Biología Pesquera. Departamento de Ciencias del Mar, Universidad Arturo Prat, Iquique, 34 pp. 
Ojeda, F.P. \& F. Kaksic. 1979. Utilización diferencial de recursos alimenticios por dos poblaciones simpatricas de Scomber japonicus peruanus y Sarda sarda chilensis en el norte de Chile. Medio Ambiente, 4(1): 19-23.

OLDEPESCA/SELA 1986. Bases biológicas y marco conceptual para el manejo de los recursos pelágicos en el Pacífico Suroriental. Publicación sobre los resultados del proyecto regional de evaluación de los recursos sardina, jurel y caballa en el $\mathrm{Pa}$ cífico Suroriental, ejecutado por Ecuador, Perú y Chile, con el apoyo financiero del Banco Interamericano de Desarrollo BID. Edición coordinada por O.A. Maithisen \& I. Tsukayama. OLDEPESCA. Documento de Pesca 1: $196 \mathrm{pp}$.

Odum, E.P. 1969. The strategy of ecosystem develompent. Science, 104: 262-270.

Odum, E.P. 1971. Fundamentals of ecology. W.B. Saunders, Philadelphia, 574 pp.

Oliva, E., M. Brown \& H. Arancibia. 1987. Alimentación de la sardina (Sardinops sagax) (Pisces: Clupeiformes), en el norte de Chile. Anales Científicos, Segundo Congreso Latinoamericano sobre Ciencias del Mar, Lima, Perú, II: 323-332.

Olson, R.J. \& G.M. Watters. 2003. Un modelo del ecosistema pelágico en el Océano Pacífico oriental tropical. Com. Interamer. Atún Trop., 22(3): 218 pp.

Ortiz, M. \& M. Wolff. 2002. Trophic models of four benthic communities in Tongoy Bay (Chile): comparative analysis and preliminary assessment of management strategies. J. Exp. Mar. Biol. Ecol., 268: 205-235.

Palomares, M.L.D. \& D. Pauly. 1989. A multiple regression model for predicting the food consumption of marine fish populations. Aust. J. Mar. Freshwater Res., 40: 259-273.

Pauly, D., A.Ch. de Vildoso, J. Mejía, M. Samamé \& M.L. Palomares. 1987. Population dynamics and estimated anchoveta consumption of bonito (Sarda chilensis) off Perú, 1953 to 1982. En: D. Pauly \& I. Tsukayama (eds.). The peruvian anchoveta and its upwelling ecosystem: three decades of change. ICLARM Studies and Reviews 15, 351 p. IMARPE, Callao, Perú; GTZ Federal Republic of Germany; and ICLARM, Manila Philippines, pp. 248-267.

Pauly, D. 1989. Food consumption by tropical and temperate fish populations: some generalizations. J. Fish. Biol., 35(Suppl. A): 11-20.

Pauly, D. \& V. Christensen. 1995. Primary produc- tion required to sustain global fisheries. Nature, 374: 255-257.

Pauly, D., A.W. Trites, E. Capuli \& V. Christensen. 1998. Diet composition and trophic levels of marine mammals. ICES J. Mar. Sci., 55: 467-481.

Pizarro, O., S. Hormazábal, A. González \& E. Yáñez. 1994. Variabilidad del viento, nivel del mar y temperatura en la costa norte de Chile. Invest. Mar., Valparaíso, 22: 83-101.

Polovina, J.J. 1984. Model of a coral ref. ecosystem I. The Ecopath model and its application to French Frigate Shoals. Coral Reefs, 3(1): 1-11.

Rice, J.C. 2000. Evaluating fishery impacts using metrics of community structure. ICES J. Mar. Sci., 57: 682-688.

Servicio Nacional de Pesca (SERNAPESCA). 1989. Anuario estadístico de pesca. Departamento de Información y Estadísticas Pesqueras. Ministerio de Economía, Fomento y Reconstrucción, Chile, 184 pp.

Sielfeld, W. 1997. Monitoreo de la pesquería y censo del lobo marino común en el litoral de la I-IV Regiones. Informe Final Proyecto FIP 95-28: 102 pp.

Shannon, L.J. \& A. Jarre-Teichmann. 1999. A model of trophic flows in the northern Benguela upwelling system during the 1980s. S. Afr. J. Mar. Sci., 21: 349-366.

Shannon L.J., P. Cury \& A. Jarre. 2000. Modelling effects of fishing in the Southern Benguela ecosystem. ICES J. Mar. Sci., 57: 720-722.

Ulanowicz, R.E. 1986. Growth and development: ecosystem phenomenology. Springer Verlag, New York, 203 pp.

Ulanowicz, R.E. 1995. The part-whole relation in ecosystems. En: B.C. Patten \& S.E. Jorgensen (eds.). Complex Ecology. Prentice-Hall, New Jersey, pp. 549-560.

Walters, C., V. Christensen \& D. Pauly. 1997. Structuring dynamic models of exploited ecosystems from trophic mass-balance assessments. Rev. Fish. Biol. Fisher., 7: 139-172.

Wolff, M. \& A. Aron. 1992. Diagnóstico de la cojinoba (Seriolella violacea) y de la palometa (Seriola mazatlana) en la IV Región. Informe Final. Primera Etapa. Universidad Católica del Norte, Sede Coquimbo, 72 pp.

Wolff, M. 1994. A trophic model for Tongoy Bay a system exposed to suspend scallop culture (Northen Chile). J. Exp. Mar. Biol. Ecol., 182: 194168 . 